\title{
THE 3-MODULAR DECOMPOSITION NUMBERS OF THE SPORADIC SIMPLE SUZUKI GROUP
}

\author{
CHRISTOPH JANSEN AND JÜRGEN MÜLLER
}

\begin{abstract}
The purpose of this paper is to determine the 3-modular decomposition numbers for the sporadic simple Suzuki group Suz. The results are obtained by a combined use of character theory, explicit construction of modules, and condensation techniques with help of the computer algebra systems MOC, MeatAxe, TensorCondense, and GAP.
\end{abstract}

\section{INTRODUCTION}

In the last years many of the decomposition matrices for the sporadic simple Suzuki group Suz have been calculated. The Brauer trees for all primes and all blocks of cyclic defect have been determined by G. Hiss and K. Lux [6]. The 2modular and 5-modular decomposition numbers are also known, although proofs have not yet been published. In this paper we determine the 3-modular decomposition numbers for Suz. Our results are collected in Section 2. Especially, it turns out that the decomposition matrix for the principal block of $S u z$ is not of wedge shape, i. e., there are no permutations of columns and rows such that the resulting matrix is lower triangular. To obtain our results we make substantial use of the computer algebra systems MOC [5], MeatAxe [13], [14], and GAP [16].

The MOC system has been developed for calculations with ordinary and Brauer characters, details can be found in [5]. Although a huge amount of data is produced in the course of our computations with MOC, using the protocol MOC provides about its actions we are able to give explicit proofs of our results, which can in principle be checked by hand. Most of the details presented in Sections 4 and 5 are obtained this way. We assume the reader to be acquainted with the notion of basic sets of projective characters and of Brauer characters, as well as with the notion of projective atoms and Brauer atoms. The strategy of the proof is as follows. We start with a basic set of Brauer characters consisting of ordinary characters restricted to 3-regular classes and a basic set of projective characters. These are then modified in a series of steps, using linear dependencies amongst Brauer characters and projective characters, respectively, finally giving a best possible approximation to the irreducible Brauer characters and the projective indecomposable characters. We use the matrix of scalar products between Brauer characters and projective characters as the leading theme in the description of the single steps, as it contains the necessary information in a most comprised form and is of wedge shape very soon.

Projective characters are obtained by induction of projective indecomposable characters of a few maximal subgroups. The necessary results for these subgroups

Date: November 12, 2002.

1991 Mathematics Subject Classification. 20C20, 20C34, $20 \mathrm{C} 40$. 
are restated in Section 3. As the irreducible Brauer characters of Suz exhaust all of the irreducible Brauer characters of the triple cover $3 \cdot S u z$ and there is a bijection between the 3-blocks of $S u z$ and those of $3 \cdot S u z$, see [4], Lemma V.4.5., it is customary to use the faithful ordinary characters of $3 \cdot S u z$ to find a suitable basic set of Brauer characters to begin with and to find relations to refine the current basic sets. In the sequel, we use faithful and non-faithful ordinary characters without further notice.

In addition to these purely character theoretic methods, we also make use of the Benson-Carlson Theorem, see [2]. These methods are sufficient then to complete the proof for the second block, whereas for the principal block a few questions remain open. This is where explicit construction and analysis of modules comes into play. The proof for the principal block is completed in Sections 7 and 8 by an analysis of several matrix and permutation representations. We make use of the Aachen implementation of the MeatAxe [14] which also includes the Lattice package [10] for the computation of submodule lattices. We assume the reader to be familiar with the standard MeatAxe techniques and the ideas introduced in [10]. We also apply fixed point condensation, which is described briefly in Section 6. Especially, we make use of the TensorCondense package [17], [11], the idea of which has already been described in [12], and which in the meantime has been incorporated into the MeatAxe [14].

All the ordinary and Brauer character tables we use can be found in [3] and [8] as far as ordinary tables and Brauer tables are concerned, respectively. These tables can also be accessed in the computer algebra system GAP. We follow the enumeration of characters, blocks, and conjugacy classes given in [3] and [8], which is the same as is provided by GAP. We also use the algorithms implemented in GAP dealing with permutation groups and character tables, e. g., to find subgroup fusions. As usual, we denote characters by their degrees, supplemented by subscripts if necessary. Note that we count each pair of faithful characters in $3 \cdot S u z$ only once.

We would like to remark that it is possible to give a complete but more complicated lengthy proof avoiding the module theoretic methods, which uses results for $2 \cdot S u z: 2$ instead. It can be found in [7]. Furthermore, as our results describe all of the irreducible Brauer characters of the triple cover $3 \cdot S u z$, the decomposition numbers for $3 \cdot S u z$ are easily found using the given results; the details are omitted. Especially, while the principal block of $3 \cdot S u z$ exactly contains the irreducible Brauer characters belonging to the principal block of $S u z$, it also contains faithful ordinary characters besides the ordinary characters belonging to the principal block of $S u z$. It can be shown that the decomposition matrix for the principal block of $3 \cdot S u z$ in fact is of wedge shape.

\section{ACKNOWLEDGEMENTS}

We wish to thank Lehrstuhl D für Mathematik, RWTH Aachen and IWR, Universität Heidelberg for providing the necessary computer equipment and Deutsche Forschungsgemeinschaft for financial support in the framework of the research project 'Algorithmic Algebra and Number Theory', which this note is a contribution to. We also wish to thank M. Schönert whose Todd-Coxeter coset enumeration program we have been allowed to use. 
TABLE 1. Decomposition matrix for the principal block

\begin{tabular}{|c|c|c|c|c|c|c|c|c|c|c|c|c|}
\hline 1 & 1 & & & & . & & $\cdot$ & & & & & \\
\hline 143 & 1 & 1 & 1 & . & . & 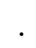 & . & . & . & & . & . \\
\hline 364 & & & 1 & 1 & . & & . & . & . & & & . \\
\hline 780 & 1 & 1 & 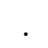 & 1 & 1 & & 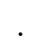 & 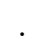 & . & & 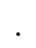 & . \\
\hline 1001 & 2 & 1 & $\cdot$ & 1 & . & 1 & 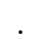 & . & 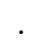 & & & . \\
\hline 3432 & 2 & 2 & . & 1 & 1 & 1 & 1 & 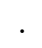 & 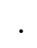 & & & . \\
\hline $5005_{1}$ & 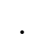 & 1 & 1 & & . & & 1 & 1 & 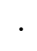 & & & . \\
\hline $5005_{2}$ & . & 1 & 1 & . & . & 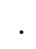 & 1 & 1 & . & & . & . \\
\hline 5940 & 2 & 1 & 1 & 4 & 1 & 2 & . & 1 & & & & \\
\hline 10725 & . & 1 & 1 & 1 & 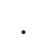 & 1 & 1 & 1 & 1 & & . & . \\
\hline 12012 & . & . & 2 & 2 & . & 1 & 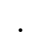 & 2 & 1 & & . & . \\
\hline 14300 & 2 & 1 & 1 & 1 & . & 1 & . & . & 1 & & . & . \\
\hline $15015_{1}$ & 2 & 1 & 1 & 2 & 1 & 1 & . & . & 1 & 1 & . & . \\
\hline $15015_{2}$ & 2 & 1 & 1 & 2 & 1 & 1 & . & . & 1 & 1 & . & . \\
\hline $25025_{1}$ & 3 & 2 & 1 & 2 & 1 & 1 & . & 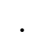 & $\cdot$ & 1 & 1 & . \\
\hline $25025_{2}$ & $\cdot$ & 1 & . & . & . & 1 & 1 & 1 & . & & . & 1 \\
\hline $25025_{3}$ & . & 1 & $\cdot$ & ${ }^{\circ}$ & . & 1 & 1 & 1 & 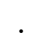 & & . & 1 \\
\hline 40040 & 5 & 4 & 3 & 5 & 1 & 3 & 1 & 2 & 1 & 1 & 1 & . \\
\hline $50050_{1}$ & 3 & 3 & 1 & 2 & 1 & 2 & 1 & 1 & $\cdot$ & 1 & 1 & 1 \\
\hline $50050_{2}$ & 3 & 3 & 1 & 2 & 1 & 2 & 1 & 1 & . & 1 & 1 & 1 \\
\hline 54054 & . & $\cdot$ & 2 & ${ }^{\circ}$ & . & & $\cdot$ & 1 & 2 & 1 & & . \\
\hline 64064 & 3 & 4 & 3 & 4 & 1 & 3 & 2 & 3 & 1 & 1 & 1 & 1 \\
\hline $64350_{1}$ & 5 & 4 & 2 & 3 & 1 & 3 & 1 & 1 & 1 & 2 & 1 & 1 \\
\hline $64350_{2}$ & 5 & 4 & 2 & 3 & 1 & 3 & 1 & 1 & 1 & 2 & 1 & 1 \\
\hline 66560 & 2 & 2 & 1 & 2 & 2 & 2 & 1 & 2 & 1 & 2 & 1 & 1 \\
\hline 75075 & 5 & 5 & 3 & 4 & 1 & 4 & 2 & 2 & 2 & 2 & 1 & 1 \\
\hline 79872 & 4 & 4 & 3 & 4 & 2 & 3 & 1 & 1 & 1 & 2 & 2 & 1 \\
\hline 100100 & 4 & 4 & 4 & 4 & 1 & 2 & 1 & 2 & 2 & 2 & 2 & . \\
\hline 133056 & 3 & 6 & 2 & 2 & 1 & 4 & 4 & 4 & 1 & 2 & 2 & 3 \\
\hline 146432 & 4 & 6 & 3 & 3 & . & 4 & 3 & 3 & 2 & 2 & 2 & 2 \\
\hline 163800 & 7 & 8 & 6 & 7 & 2 & 5 & 3 & 5 & 3 & 3 & 3 & 1 \\
\hline 168960 & 6 & 8 & 4 & 5 & 2 & 5 & 3 & 2 & 2 & 3 & 3 & 2 \\
\hline 193050 & 5 & 7 & 5 & 6 & 2 & 6 & 4 & 6 & 3 & 3 & 2 & 3 \\
\hline 197120 & 8 & 8 & 6 & 7 & 2 & 6 & 3 & 5 & 4 & 4 & 3 & 2 \\
\hline
\end{tabular}

\section{The Results}

2.1. The Principal Block. The principal block consists of 34 ordinary and 13 Brauer characters. Its decomposition matrix is given in Table 1. The Brauer characters are given as follows.

$$
\{1,64,78,286,429,649,1938,2925,4785,8436,14730,19449,32967\}
$$

2.2. The Second Block. The second block consists of 6 ordinary characters and 5 Brauer characters. Its decomposition matrix can be found in Table 2. The Brauer 
TABle 2. Decomposition matrix for the second block

\begin{tabular}{r|ccccc}
15795 & 1 &. &. &. &. \\
88452 & 1 & 1 &. &. &. \\
$93555_{1}$ & 1 & 1 & 1 &. &. \\
$93555_{2}$ & 1 & 1 &. & 1 &. \\
243243 &. & 1 & 1 & 1 & 1 \\
248832 & 1 & 1 &. &. & 1
\end{tabular}

TABLE 3. Decomposition matrix for the third block

\begin{tabular}{r|cc}
18954 & 1 &. \\
189540 & $\cdot$ & 1 \\
208494 & 1 & 1
\end{tabular}

characters are given as follows.

$$
\left\{15795,72657,5103_{1}, 5103_{2}, 160380\right\}
$$

2.3. The Third Block. The third block consists of 3 ordinary characters, namely 18954,189540 , and 208494 , it is of cyclic defect. The decomposition matrix can be found in [6], we state it for convenience in Table 3.

\section{Modular Tables For Some Maximal Subgroups}

We make use of the ordinary character tables of four of the maximal subgroups of $S u z$, namely $G_{2}(4), U_{5}(2), 2_{-}^{1+6} \cdot U_{4}(2)$, and $2^{4+6}: 3 A_{6}$, and of the 3 -modular Brauer character tables of $G_{2}(4), U_{5}(2), U_{4}(2)$, and $A_{6}$. As it is not a priori clear that the subgroup fusions of these subgroups into $S u z$ provided by GAP are compatible with each other and with the Brauer character tables, we have to check this. It turns out that there are only ambiguities concerning conjugacy classes of 3 -singular elements and additionally one concerning classes of elements of order 13 in $G_{2}(4)$. Hence we are free to choose the subgroup fusions as they are provided by GAP.

Let us now fix some notation. The irreducible Brauer characters of the subgroups mentioned above are denoted by $\varphi_{i}$, where we follow the enumeration given in [8] and GAP. The ordinary character of the projective cover of such an irreducible Brauer character $\varphi$ is denoted by $\Phi(\varphi)$. We then obtain projective characters $\Phi \uparrow^{S u z}$ of $S u z$ by induction from the projective indecomposable characters $\Phi$ of these subgroups. The induced projective characters are denoted by small letter $\phi$ 's, supplemented by a suitable index. Later on, we restrict the $\phi$ 's to the respective blocks being under consideration.

3.1. $G_{2}(4)$. Let $\left\{\phi_{1}, \ldots, \phi_{17}\right\}$ be the following induced characters

$$
\begin{gathered}
\Phi\left(\varphi_{13}\right) \uparrow^{S u z}, \Phi\left(\varphi_{7}\right) \uparrow^{S u z}, \Phi\left(\varphi_{4}\right) \uparrow^{S u z}, \Phi\left(\varphi_{5}\right) \uparrow^{S u z}, \Phi\left(\varphi_{3}\right) \uparrow^{S u z}, \Phi\left(\varphi_{2}\right) \uparrow^{S u z}, \\
\Phi\left(\varphi_{1}\right) \uparrow^{S u z}, \Phi\left(\varphi_{15}\right) \uparrow^{S u z}, \Phi\left(\varphi_{8}\right) \uparrow^{S u z}, \Phi\left(\varphi_{9}\right) \uparrow^{S u z}, \Phi\left(\varphi_{16}\right) \uparrow^{S u z}, \Phi\left(\varphi_{10}\right) \uparrow^{S u z}, \\
\Phi\left(\varphi_{17}\right) \uparrow^{S u z}, \Phi\left(\varphi_{12}\right) \uparrow^{S u z}, \Phi\left(\varphi_{6}\right) \uparrow^{S u z}, \Phi\left(\varphi_{18}\right) \uparrow^{S u z}, \Phi\left(\varphi_{19}\right) \uparrow^{S u z} .
\end{gathered}
$$


3.2. $U_{5}(2)$. We take $\phi_{18}:=\Phi\left(\varphi_{9}\right) \uparrow^{S u z}$.

3.3. $2_{-}^{1+6} \cdot U_{4}(2)$. Let $\varphi_{1}$ be the the trivial character of $2_{-}^{1+6} \cdot U_{4}(2)$. By [4], Lemma V.4.3., $\Phi\left(\varphi_{1}\right)$ only consists of characters belonging to the factor group $U_{4}(2)$, whose 3 -modular table is known. As $2_{-}^{1+6}$ is the maximal 2-normal subgroup, the factor fusion from $2_{-}^{1+6 \cdot} U_{4}(2)$ to $U_{4}(2)$ is uniquely determined. This determines $\Phi\left(\varphi_{1}\right)$ and we take $\phi_{19}:=\Phi\left(\varphi_{1}\right) \uparrow$ Suz .

3.4. $2^{4+6}: 3 A_{6}$. We only need projective indecomposable characters belonging to blocks which have the maximal 2-normal subgroup $2^{4+6}$ in their kernel. Hence again by [4], Lemma V.4.3., we are content with the 3-modular decomposition matrix of $3 A_{6}$, which in turn is determined by the 3 -modular Brauer character table of $A_{6}$. Let $\phi_{20}:=\Phi\left(\varphi_{1}\right) \uparrow^{S u z}, \phi_{21}:=\Phi\left(\varphi_{4}\right) \uparrow^{S u z}, \phi_{22}:=\Phi\left(\varphi_{5}\right) \uparrow^{S u z}$.

\section{The Principal Block}

4.1. First of all we form a basic set of Brauer characters for the principal block, consisting of ordinary characters of $3 \cdot \mathrm{Suz}$ restricted to the 3 -regular classes. We choose

$$
B S:=\left\{1,66,78,364,429,1001,2145,2925_{1}, 10725,14300,24024,25025_{2}, 54054\right\} .
$$

This is indeed a basic set of Brauer characters as it can be proved that the restrictions to the 3-regular classes of the ordinary characters belonging to the principal block decompose into $B S$ with integral coefficients. Next we let $P S:=$ $\left\{\psi_{1}, \ldots, \psi_{13}\right\}$ where the $\psi_{i}$ are the restrictions of

$$
\phi_{16}, \phi_{8}, \phi_{11}, \phi_{18}, \phi_{12}, \phi_{14}, \phi_{9}, \phi_{2}, \phi_{4}, \phi_{3}, \phi_{5}, \phi_{6}, \phi_{7} \text {, }
$$

respectively, to the principal block. This is a basic set of projective characters, since it can be shown that the elementary divisors of the matrix of scalar products with $B S$ are all equal to 1 .

4.2. In the sequel we denote by $B A$ and $P A$ the bases dual to the current bases $P S$ and $B S$ with respect to taking scalar products. Then the projective characters $\psi_{1}, \ldots, \psi_{5}$ decompose into $P A$ as follows.

$$
\begin{aligned}
& \psi_{1}=P A \cdot(0,0,0,0,0,0,0,0,0,0,0,1,1)^{t}, \\
& \psi_{2}=P A \cdot(0,0,0,0,0,0,0,0,0,0,1,1,0)^{t}, \\
& \psi_{3}=P A \cdot(0,0,0,0,0,0,0,0,0,1,0,0,3)^{t}, \\
& \psi_{4}=P A \cdot(0,0,0,0,0,0,0,0,0,1,1,3,4)^{t}, \\
& \psi_{5}=P A \cdot(0,0,0,0,0,0,0,0,1,1,0,0,3)^{t} .
\end{aligned}
$$

Let $\psi_{14}$ be the restriction of $\phi_{1}$ to the principal block. Then $\psi_{14}$ decomposes into $P S$ as $\psi_{14}=\psi_{5}+2 \cdot \psi_{2}-\psi_{1}$. Therefore $\psi_{1}$ cannot be indecomposable, hence $\psi_{15}:=-\psi_{1}-\psi_{2}-\psi_{3}+\psi_{4}$ and $\psi_{16}:=\psi_{1}-\psi_{15}$ are projective. We set $P S$ as follows, the corresponding matrix of scalar products being given in Table 4,

$$
P S:=\left\{\psi_{16}, \psi_{15}, \psi_{2}, \psi_{3}, \psi_{5}, \ldots, \psi_{13}\right\} .
$$


TABLE 4. First matrix of scalar products

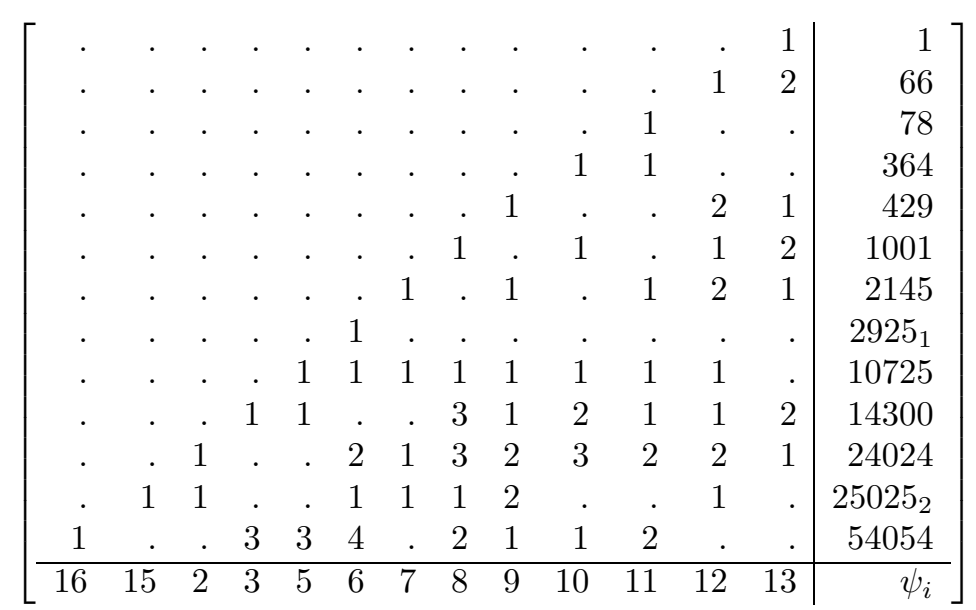

TABLE 5. Second matrix of scalar products

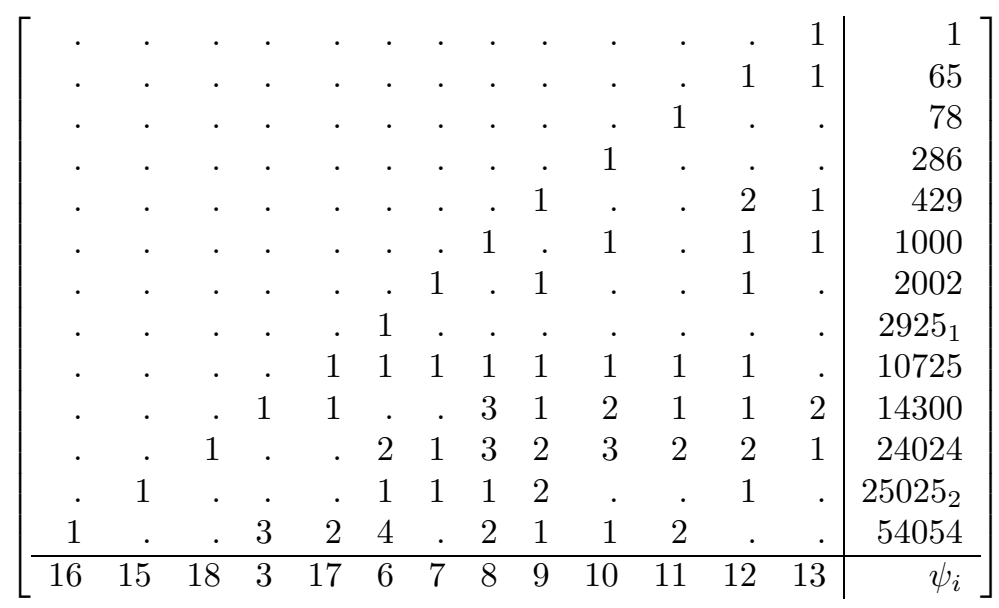

4.3. Now we have the following relation $\psi_{14}=-\psi_{16}-\psi_{15}+2 \cdot \psi_{2}+\psi_{5}$, hence $\psi_{17}:=\psi_{5}-\psi_{16}$ and $\psi_{18}:=\psi_{2}-\psi_{15}$ are projective.

From $143=-1+66+78$ on 3 -regular classes we deduce that $65:=66-1$ is a Brauer character. From $1716=-78+364+429+1001$ we find $286:=364-78$, and from $23100=-1+1001-2925_{1}+25025_{2}$ we find $1000:=1001-1$ as Brauer characters. Using $5005_{1}=1-66+2145+2925_{1}$ and $3432=1-66-78+429+$ $1001+2145$ we finally get the existence of $2002:=2145-78-65$. This gives us new basic sets, whose matrix of scalar products is given in Table 5 ,

$$
P S:=\left\{\psi_{16}, \psi_{15}, \psi_{18}, \psi_{3}, \psi_{17}, \psi_{6}, \ldots, \psi_{13}\right\},
$$

$B S:=\left\{1,65,78,286,429,1000,2002,2925_{1}, 10725,14300,24024,25025_{2}, 54054\right\}$. 
TABLE 6. Third matrix of scalar products

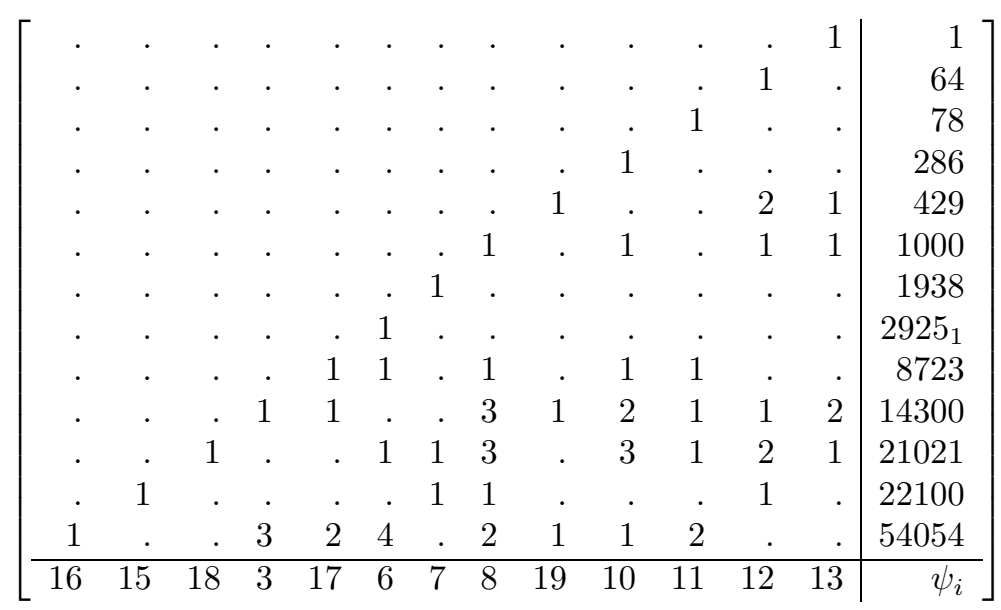

4.4. Now 12012 decomposes into $B S$ as $12012=78+286-2002+2925_{1}+10725$. As 78,286 and $2925_{1}$ are irreducible Brauer characters and cannot be contained in 2002 we get that $8723:=10725-2002$ is a Brauer character. Next $21450=$ $-78+429-2925_{1}+24024$ proves the existence of $21021:=24024-78-2925_{1}$. Using $23100=1000-2925_{1}+25025_{2}$ we see that $22100:=25025_{2}-2925_{1}$ is a Brauer character.

4.5. Now we consider the 12-dimensional self-dual representation of $2 \cdot S u z$ over $G F(3)$, which is irreducible, since the same is true for its restriction to $2 \cdot G_{2}(4)$. The tensor product $12 \otimes 12$ is also self-dual and, using the Benson-Carlson Theorem [2], see also [1] 3.1.9., each direct summand has a dimension divisible by 3 . The tensor product decomposes into its symmetric and antisymmetric parts as $12 \otimes 12=$ $12^{(2+)} \oplus 12^{(2-)}$, where $12^{(2-)}=65+1$ as Brauer characters. By looking at the matrix of scalar products we see that either 65 is irreducible or has to be decomposed into $64+1$. Hence $12^{(2-)}$ is uniserial with ascending composition series $1,64,1$, and $64:=65-1$ is a Brauer character.

Furthermore we have $64^{(2-)}=-64+78+2002$, this proves the existence of $1938:=2002-64$. We obtain the following basic set of Brauer characters

$$
B S:=\{1,64,78,286,429,1000,1938,29251,8723,14300,21021,22100,54054\} .
$$

Let $\psi_{19}$ be the restriction of $\phi_{22}$ to the principal block. The following can be shown to be a basic set using the matrix of scalar products given in Table 6 ,

$$
P S:=\left\{\psi_{16}, \psi_{15}, \psi_{18}, \psi_{3}, \psi_{17}, \psi_{6}, \psi_{7}, \psi_{8}, \psi_{19}, \psi_{10}, \psi_{11}, \psi_{12}, \psi_{13}\right\} .
$$

4.6. We consider the restrictions $\psi_{20}$ and $\psi_{21}$ of $\phi_{20}$ and $\phi_{21}$, respectively, to the principal block. They fulfill the following relations $\psi_{20}=-\psi_{19}+\psi_{10}+\psi_{13}$ and $\psi_{21}=\psi_{8}-2 \cdot \psi_{19}+\psi_{12}$. Hence the Brauer character 429 must be irreducible.

Furthermore, $\psi_{20}$ proves that $\psi_{19}$ cannot be indecomposable as $\psi_{13}-\psi_{19}$ is not projective. Therefore, $\psi_{19}$ has to be decomposed. The decompositions

$$
P A \cdot((0,0,0,0,1,0,0,0,0,1,0,0,0)+(0,0,0,0,0,0,0,0,0,0,0,0,1))^{t} \text { and }
$$


TABLE 7. Fourth matrix of scalar products

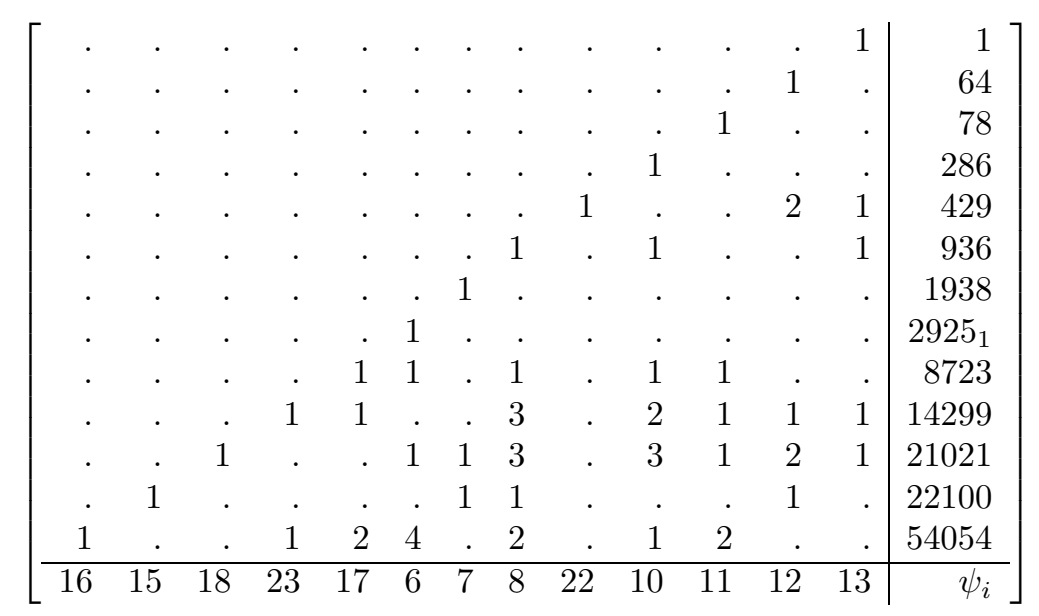

$P A \cdot\left(((0,0,0,0,1,0,0,0,0,0,0,0,1)+(0,0,0,0,0,0,0,0,0,1,0,0,0))^{t}\right.$

cannot be correct because of $\psi_{21}$. Therefore we have found

$$
\begin{gathered}
\psi_{22}:=P A \cdot(0,0,0,0,1,0,0,0,0,0,0,0,0)^{t} \text { and } \\
\psi_{23}:=P A \cdot(0,0,0,0,0,0,0,0,0,1,0,0,1)^{t}
\end{gathered}
$$

as new projective characters.

Because of $5940=-64+78+2 \cdot 286+429+2 \cdot 1000+2925_{1}$ we have $936:=1000-64$ as a Brauer character. Similarly, using the irreducibility of 429 and the relation $66560=-2 \cdot 1-2 \cdot 64-78+2 \cdot 429-1000-1938+2 \cdot 2925_{1}-8723+2 \cdot 14300+$ $21021+22100$, we find $14299:=14300-1$ to be a new Brauer character. This leads to new basic sets with matrix of scalar products in Table 7 ,

$$
P S:=\left\{\psi_{16}, \psi_{15}, \psi_{18}, \psi_{23}, \psi_{17}, \psi_{6}, \psi_{7}, \psi_{8}, \psi_{22}, \psi_{10}, \psi_{11}, \psi_{12}, \psi_{13}\right\},
$$

$$
B S:=\left\{1,64,78,286,429,936,1938,2925_{1}, 8723,14299,21021,22100,54054\right\} .
$$

4.7. Now $\psi_{20}=-\psi_{23}-\psi_{22}+\psi_{10}+\psi_{13}$ proves that $\psi_{24}:=\psi_{13}-\psi_{22}$ is projective. $\psi_{21}=-2 \cdot \psi_{23}+\psi_{8}-2 \cdot \psi_{22}+\psi_{12}$ shows that $\psi_{25}:=\psi_{12}-2 \cdot \psi_{22}$ and $\psi_{26}:=\psi_{8}-\psi_{23}$ are projective characters.

Since $8723-936-78+1$ is the sum of two Brauer atoms, one of which equals the irreducible Brauer character $2925_{1}$, the relation $25025_{1}=1-64+429-1938-8723+$ $14299+21021$ proves that $5798:=8723-2925_{1}$ and $16158:=21021-1938-2925_{1}$ are Brauer characters. Changing basic sets leads to

$$
P S:=\left\{\psi_{16}, \psi_{15}, \psi_{18}, \psi_{23}, \psi_{17}, \psi_{6}, \psi_{7}, \psi_{26}, \psi_{22}, \psi_{10}, \psi_{11}, \psi_{25}, \psi_{24}\right\},
$$

$B S:=\left\{1,64,78,286,429,936,1938,2925_{1}, 5798,14299,16158,22100,54054\right\}$. 
TABLE 8. Fifth matrix of scalar products

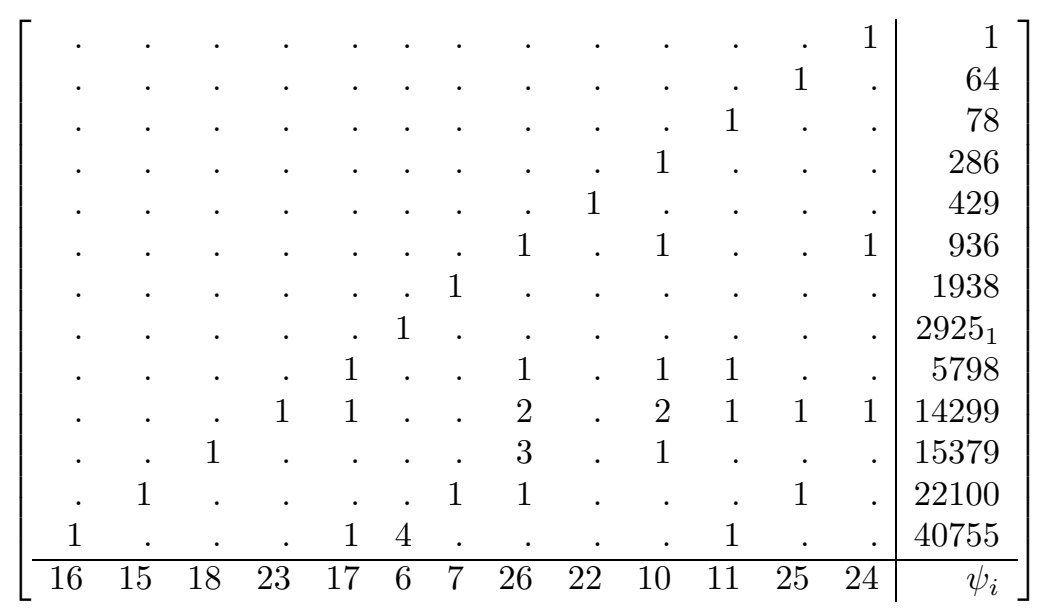

4.8. We now consider some tensor products between Brauer characters. We have $64 \otimes 286=-1-2 \cdot 64-78-2 \cdot 286+2925_{1}+16158$. Therefore $15379:=16158-$ $1-2 \cdot 64-78-2 \cdot 286$ is a Brauer character, as $2925_{1}$ is irreducible. Now let $40755:=286^{(2-)}=64+936-14299+54054$. We change $B S$ again, obtaining the new matrix of scalar products in Table 8 ,

$B S:=\left\{1,64,78,286,429,936,1938,2925_{1}, 5798,14299,15379,22100,40755\right\}$.

4.9. Considering $54054=-64-936+14299+40755$, we find that $13299:=$ $14299-936-64$ is a Brauer character.

From $\psi_{20}=-\psi_{23}+\psi_{10}+\psi_{24}$ we see that $\psi_{27}:=\psi_{10}-\psi_{23}$ is projective, and from $\psi_{21}=-\psi_{23}+\psi_{26}+\psi_{25}$ we conclude that the character $\psi_{28}:=\psi_{26}-\psi_{23}$ is projective.

Using the relation $25025_{1}=2 \cdot 1+2 \cdot 64+78+2 \cdot 286+429+936-5798+13299+15379$ and the decomposition $13299=(0,0,0,1,1,0,0,0,0,0,1,0,0) \cdot B A$, it can be shown that $4863:=5798-936+1$ and $8514:=13299-4863+78$ are Brauer characters. Changing basic sets gives, with corresponding matrix of scalar products in Table 9 ,

$$
\begin{gathered}
P S:=\left\{\psi_{16}, \psi_{15}, \psi_{18}, \psi_{23}, \psi_{17}, \psi_{6}, \psi_{7}, \psi_{28}, \psi_{22}, \psi_{27}, \psi_{11}, \psi_{25}, \psi_{24}\right\}, \\
B S:=\left\{1,64,78,286,429,936,1938,2925_{1}, 4863,8514,15379,22100,40755\right\} .
\end{gathered}
$$

4.10. Using $5798=-1+936+4863$ we get that $935:=936-1$ is a Brauer character. $60060=3 \cdot 1+2 \cdot 64-2 \cdot 78+286+429+4863+2 \cdot 8514+15379+22100$ proves that $8436:=8514-78$ is a Brauer character. Since we find $64 \otimes 935=$ $2 \cdot 1+2 \cdot 286-935-1938+2925_{1}+4863+2 \cdot 8436+15379+22100$ we get the existence of $20162:=22100-1938$. We now have the following basic set,

$$
B S:=\left\{1,64,78,286,429,935,1938,2925_{1}, 4863,8436,15379,20162,40755\right\} .
$$


TABLE 9. Sixth matrix of scalar products

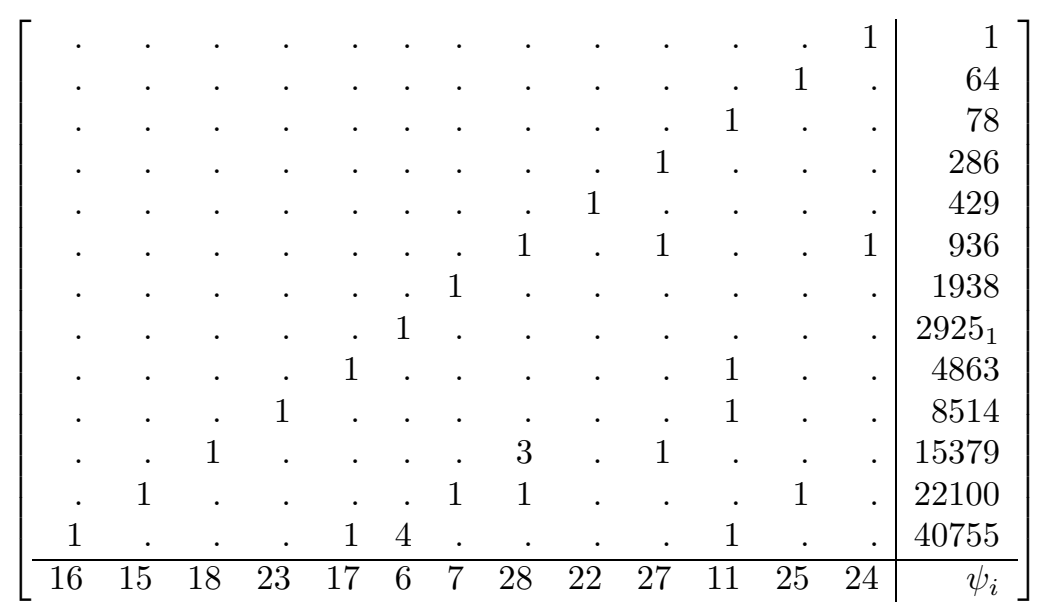

TABLE 10. Seventh matrix of scalar products

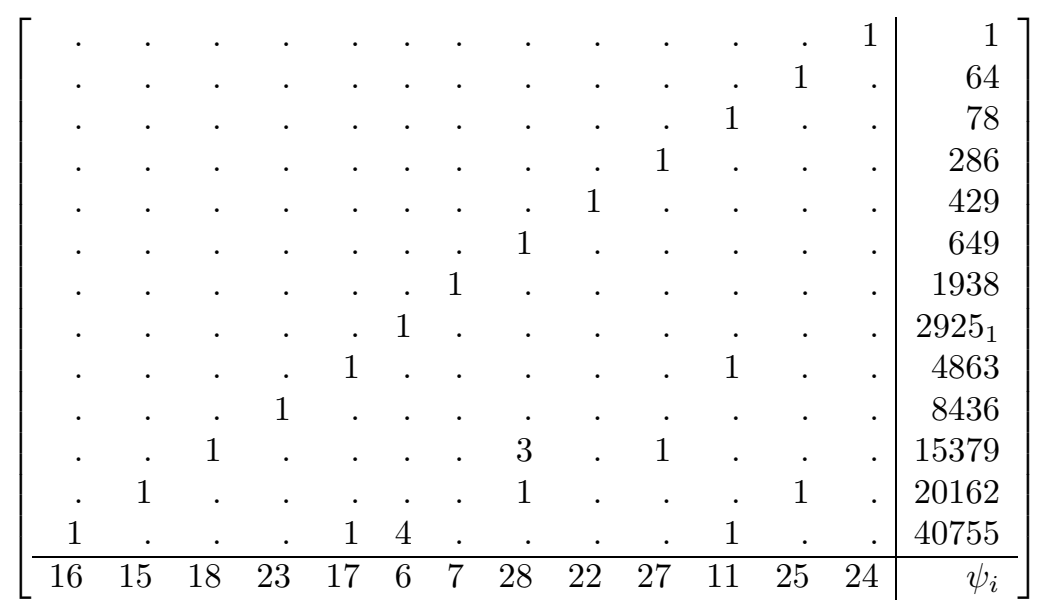

4.11. The only remaining question concerning 935 is, whether it is irreducible or $649:=935-286$ is an irreducible Brauer character. In Section 8.1 we show that 649 exists. We replace the basic set, getting the scalar products in Table 10,

$$
B S:=\left\{1,64,78,286,429,649,1938,2925_{1}, 4863,8436,15379,20162,40755\right\} .
$$

4.12. Now assume that 4863 is irreducible. Using the relations $78 \otimes 649=-649-$ $4863+15379+40755$ and $649^{(2-)}=-78+1938-2925_{1}+40755+\vartheta$, where $\vartheta$ denotes the component of $649^{(2-)}$ belonging to the non-principal blocks, we see that 40755 contains 4863 and 78. This is a contradiction to the decomposition of 40755 into atoms as can be seen from the matrix of scalar products given in Table 10. Therefore $4785:=4863-78$ exists. 
TABLE 11. Eighth matrix of scalar products

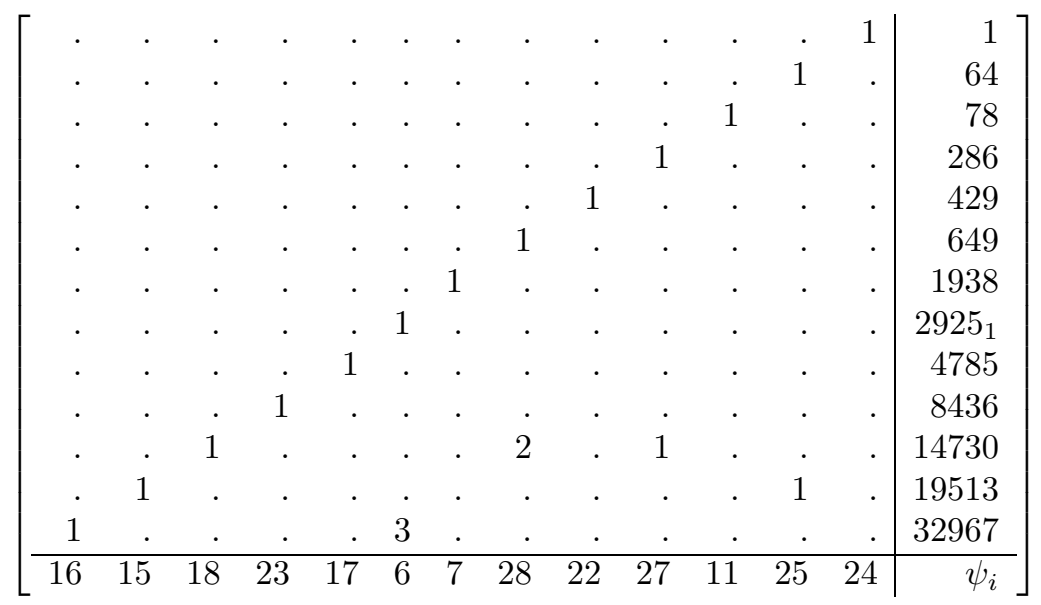

Then we get that $32967:=40755-78-2925_{1}-4785$ is a Brauer character. Also due to $78 \otimes 649$ we conclude that $14730:=15379-649$ is a Brauer character. When considering $64 \otimes 649=2 \cdot 1+78+286-649+4785+2 \cdot 8436+20162$ we conclude that $19513:=20162-649$ is a Brauer character. We take the new basic set as follows, with matrix of scalar products as it is in Table 11,

$$
B S:=\left\{1,64,78,286,429,649,1938,2925_{1}, 4785,8436,14730,19513,32967\right\} .
$$

4.13. Hence we know all the irreducible Brauer characters in the principal block except $\varphi_{11}, \varphi_{12}$ and $\varphi_{13}$. There are the following possible cases left. $\varphi_{11}=14730-$ $x \cdot 286-y \cdot 649$ where $x \in\{0,1\}$ and $y \in\{0,1,2\}, \varphi_{12}=19513-z \cdot 64$ where $z \in\{0,1\}$, and $\varphi_{13}=32967-w \cdot 2925_{1}$ where $w \in\{0,1,2,3\}$. The values for $x, y$, $z$, and $w$ are determined in Sections 8.2, 8.3, and 8.5.

\section{The Second Block}

5.1. Let $\left\{\psi_{29}, \ldots, \psi_{33}\right\}$ be the restrictions of $\phi_{15}, \frac{1}{3} \phi_{14}, \phi_{9}, \phi_{16}$, and $\frac{1}{3} \phi_{5}$, respectively, to the second block.

Here we use the following notation. Inducing the projective character $\phi_{14}$ up to $S u z$ and restricting it to the second block yields a character whose scalar products with all the ordinary characters in this block are divisible by 3 . Hence we can divide all these by 3 and still have a projective character, which then is denoted by $\psi_{30}$. The character $\psi_{33}$ is found similarly.

Then we get $\psi_{30}+\psi_{31}=2 \cdot\left(88452+93555_{1}+93555_{2}+4 \cdot 243243+4 \cdot 248832\right)$ as ordinary characters. Hence $\psi_{34}:=\left(\psi_{30}+\psi_{31}\right) / 2$ is projective. Now we take

$$
P S:=\left\{\psi_{29}, \psi_{30}, \psi_{34}, \psi_{32}, \psi_{33}\right\}
$$

and the following ordinary characters restricted to the 3-regular classes as $B S$. By the matrix of scalar products in Table 12 these are indeed basic sets,

$$
B S:=\left\{15795,88452,160380,5103_{1}, 5103_{2}\right\} \text {. }
$$


TABLE 12. First matrix of scalar products

$$
\left[\begin{array}{rrrrr|r}
\cdot & . & . & . & 1 & 15795 \\
\cdot & 1 & 1 & 1 & 1 & 88452 \\
. & 2 & 3 & 4 & 1 & 160380 \\
1 & . & . & . & . & 5103_{1} \\
1 & . & . & 1 & . & 5103_{2} \\
\hline 29 & 30 & 34 & 32 & 33 & \psi_{i}
\end{array}\right]
$$

TABLE 13. Second matrix of scalar products

$$
\left[\begin{array}{rrrrr|r}
\cdot & . & . & . & 1 & 15795 \\
\cdot & . & . & 1 & 1 & 88452 \\
\cdot & . & 1 & . & . & 5103_{1} \\
\cdot & 1 & . & . & . & 5103_{2} \\
1 & . & . & . & 1 & 160380 \\
\hline 41 & 38 & 39 & 40 & 33 & \psi_{i}
\end{array}\right]
$$

5.2. Now let $\psi_{35}, \psi_{36}$, and $\psi_{37}$ be the restrictions of $\phi_{17}, \phi_{13}$, and $\phi_{10}$, respectively, to the second block. These decompose into $P S$ as $\psi_{35}=\psi_{29}-2 \cdot \psi_{30}+4 \cdot \psi_{34}-\psi_{32}$, $\psi_{36}=9 \cdot \psi_{30}-5 \cdot \psi_{34}$, and $\psi_{37}=-\psi_{30}+2 \cdot \psi_{34}$.

We now show that $\psi_{30}$ decomposes into projective indecomposable characters as $\psi_{30}=P A \cdot((0,1,0,0,0)+(0,0,1,0,0)+(0,0,1,0,0))^{t}$. There are three other cases to exclude. $(i) \psi_{30}$ is indecomposable. That is impossible due to $\psi_{36}$ and $\psi_{37}$. (ii) $\psi_{30}=P A \cdot((0,1,0,0,0)+(0,0,2,0,0))^{t}$. This is not correct because it implies the existence of an indecomposable projective character which is twice an atom. (iii) $\psi_{30}=P A \cdot((0,1,1,0,0)+(0,0,1,0,0))^{t}$. As $\psi_{30}+\psi_{31}=2 \cdot \psi_{34}$, we have $\psi_{34}=$ $P A \cdot((0,1,1,0,0)+(0,0,1,0,0)+(0,0,1,0,0))^{t}$, hence $\psi_{36}=P A \cdot(4 \cdot(0,1,1,0,0)-$ $1 \cdot(0,0,1,0,0))^{t}$, which is not a positive decomposition into indecomposables.

Furthermore $\psi_{35}$ proves that $\psi_{29}$ cannot be indecomposable. Now we put $\psi_{38}:=$ $P A \cdot(0,0,0,0,1)^{t}, \psi_{39}:=P A \cdot(0,0,0,1,0)^{t}, \psi_{40}:=P A \cdot(0,1,0,0,0)^{t}$, and $\psi_{41}:=$ $P A \cdot(0,0,1,0,0)^{t}$, and let, with matrix of scalar products given in Table 13 ,

$$
\begin{gathered}
P S:=\left\{\psi_{41}, \psi_{38}, \psi_{39}, \psi_{40}, \psi_{33}\right\}, \\
B S:=\left\{15795,88452,5103_{1}, 5103_{2}, 160380\right\} .
\end{gathered}
$$

5.3. Finally, let $\psi_{42}$ be the restriction of $\phi_{19}$ to the second block. Then $\psi_{42}=$ $-\psi_{41}+\psi_{40}+5 \cdot \psi_{33}$. Therefore $\psi_{43}:=\psi_{33}-\psi_{41}$ is projective. Using the relation $243243=-15795+88452+5103_{1}+5103_{2}+160380$ we see that $72657:=88452-$ 15795 is a Brauer character. This concludes the proof for the second block.

\section{Some Remarks On Condensation}

To complete the decomposition matrix for the principal block, we construct and analyze several matrix and permutation representations of Suz explicitly. We use fixed point condensation to obtain modules of a size which makes it possible to 
examine submodule structures explicitly. In this section we give a few comments and remarks concerning this method and how it is applied.

6.1. Let $F$ be a field, $H$ a subgroup of a finite group $G$, such that the characteristic of $F$ does not divide the order $|H|$. Then we have the following idempotent in the group algebra $F G$,

$$
e=e_{H}:=|H|^{-1} \cdot \sum_{h \in H} h .
$$

Now let $V$ be an $F G$-module. Then the subset $V e$ of $V$ is the set of fixed points of $V$ under the action of $H$. As $V$ is semisimple as a module for $F H$, the dimension of $V e$ can be determined by calculating the ordinary scalar product between the restriction to $H$ of the Brauer character of $V$ and the trivial character of $H$. Ve is a module for the Hecke algebra eFGe. Hence we have a condensation functor from the category of finitely generated $F G$-modules to the category of finitely generated $e F G e$-modules. The application of this functor to an $F G$-module $V$ is called fixed point condensation. A few of the properties of this functor, especially as far as the submodule structures of $V$ and $V e$ are concerned, are described in [15] or [12]. Especially, if we have $S e \neq\{0\}$ for each constituent $S$ of $V$, then the submodule lattices of $V$ and $V e$ are naturally isomorphic. The condensation functor can be efficiently computed for permutation modules of degree up to several million and for tensor product modules of dimension up to several ten thousand. Programs performing these tasks are implemented in the MeatAxe and in the TensorCondense package, which both have already been mentioned in Section 1.

6.2. Now let $\left\{g_{1}, g_{2}, \ldots, g_{n}\right\}$ be a set of generators for $G$. Then the subalgebra $C$ of $e F G e$ generated by $\left\{e g_{1} e, e g_{2} e, \ldots, e g_{n} e\right\}$ is called the condensation algebra with respect to this generating set. It is not necessarily equal to $e F G e$. Hence we have to distinguish carefully between $V e$ as a $C$-module and as an $e F G e$-module. We analyze the submodule structure of $V e$ as a $C$-module using the computational methods mentioned above, and then we have to draw conclusions about its structure as an $e F G e$-module. One example how this works is given in Remark 6.3 below, which is applied in 8.3. A more systematic approach to these problems can be found in [7].

6.3. Remark. For later use, we briefly recall the following notion from [10]. Let $R$ be a ring. An $R$-module is called local, if it has a uniquely determined maximal $R$-submodule. A local $R$-module is called $S$-local, if its head is isomorphic to the simple $R$-module $S$.

Now let $M$ be an $e F G e$-module. Let $U^{\prime}<U$ be $e F G e$-submodules of $M$, such that $S:=U / U^{\prime}$ is an irreducible $e F G e$-module, and assume further that $\tilde{S}:=\left.S\right|_{C}$ is an irreducible $C$-module. Then there is an $\tilde{S}$-local $C$-submodule $\tilde{L} \leq U$, such that $\tilde{L} \cdot e F G e \leq U$ is an $S$-local $e F G e$-submodule.

To see this, note that by the assumptions there is an $S$-local $e F G e$-submodule $L \leq U$ having the maximal eFGe-submodule $L^{\prime}<L$, such that $L+U^{\prime}=U$ and $L \cap U^{\prime}=L^{\prime}$. As $\left.S\right|_{C}$ is irreducible, there is an $\tilde{S}$-local $C$-submodule $\tilde{L} \leq L$ having the maximal $C$-submodule $\tilde{L}^{\prime}<\tilde{L}$, such that $\tilde{L}+L^{\prime}=L$ and $\tilde{L} \cap L^{\prime}=L^{\prime}$. Now we have $\tilde{L} \cdot e F G e=L$. 
TABLE 14. Permutation representations

$$
\begin{array}{l|l|r|l}
\text { Suz.p1782 } & G_{2}(4) & 1782 & 1+780+1001 \\
\text { Suz.p22880 } & 3 \cdot U_{4}(3): 2 & 22880 & 1+364+780+5940+15795 \\
\text { Suz.p135135 } & 2_{-}^{1+6 \cdot} \cdot U_{4}(2) & 135135 & 1+143+780+3432+5940+ \\
& & & 14300+18954+25025_{1}+66560
\end{array}
$$

6.4. One of the most important tools to ensure the existence of certain submodules of $V e$ as an $e F G e$-module is the following theorem. First we have to recall the following notion, which together with a few different characterisations can be found in [9], Definition I.17.1.

Let $(K, R, F)$ be a modular system for $G$, i. e., $R$ is a discrete valuation ring with fraction field $K$ and quotient field $F$, such that $K$ and $F$ are splitting fields for $G$. Let $M$ be a free $R$-module. An $R$-submodule $N \leq M$ is called $R$-pure, if the quotient $M / N$ is again free as an $R$-module. In this case, it follows that the $F$-modular reduction of $N$ is a submodule of the $F$-modular reduction of $M$.

6.5. Theorem. (Zassenhaus and others). Let $(K, R, F)$ be as above and let $M$ be an $R$-free $R G$-module with ordinary character $\chi$, such that $\chi=\chi^{\prime}+\chi^{\prime \prime}$ as ordinary characters. Then there exists an $R$-pure $R G$-submodule $N \leq M$ with character $\chi^{\prime}$.

For a proof we refer to [9], Theorem I.17.3. As the $F$-modular reduction of $N$ is a submodule of the $F$-modular reduction of $M$, this theorem ensures the existence of $F G$-submodules of $M$ whose modular constituents can be controlled.

\section{Construction Of Representations}

7.1. We now give constructions for three transitive permutation representations, Suz.p1782, Suz.p22880, and Suz.p135135, we use in the sequel. In Table 14 we write down the corresponding one point stabilizer, the degree, and the permutation character.

7.2. We start with the presentation $S u z: 2:=\langle a, b, c, d, e, f, g \mid \mathcal{R}\rangle$, where $\mathcal{R}$ is the following set of relators:

$$
\begin{gathered}
\left\{a^{2}, b^{2},(a b)^{5}, c^{2},(a c)^{2},(b c)^{3}, d^{2},(a d)^{2},(b d)^{2},(c d)^{8}, e^{2},(a e)^{2},(b e)^{2},(c e)^{2},(d e)^{3},\right. \\
f^{2},(a f)^{2},(b f)^{3},(c f)^{2},(d f)^{2},(e f)^{2}, g^{2},(a g)^{2},(b g)^{2},(c g)^{2},(d g)^{2},(e g)^{2},(f g)^{8}, \\
\left.a^{-1}(c d)^{4},(a b c d e c b d c)^{7},(a b c f)^{5}, a^{-1}(f g)^{4},(b f g f g)^{5}\right\} .
\end{gathered}
$$

This is the second presentation to be found in [3], p. 131 .

Let $A:=f$ and $B:=$ gabcfdeg. Using a Todd-Coxeter coset enumeration with respect to the subgroup $S u z:=\langle A, B\rangle$ of $S u z: 2$, we find that this subgroup has indeed index 2. Using a Todd-Coxeter coset enumeration again, now with respect to the subgroup $\left\langle a, b, c, d, e, f, h^{2} g\right\rangle$, where $h:=g f b c d c b f$, gives us a permutation representation of $S u z: 2$ on 1782 points. By restriction to $S u z$ we obtain the representation Suz.p1782. Similarly, using the subgroup $\langle a, b, c, d, f, g\rangle$ and restriction to $S u z$ we obtain the permutation representation $S u z . p 22880$.

By some abuse of notation we also denote the homomorphic images of $A$ and $B$ in several representations by the same symbols. This should not lead to any confusion. 
7.3. Our next task is to construct the permutation representation Suz.p135135. The one point stabilizer is the forth maximal subgroup $M_{4}:=2_{-}^{1+6} \cdot U_{4}(2)$, which is the centralizer of a $2 A$ element of $S u z$. Note that we have already encountered $M_{4}$ in Section 3.3. Now $A$ is of order 2, and as it has 54 fixed points in the representation Suz.p1782, it is a $2 A$ element. Let $C_{1}:=A B, C_{2}:=C_{1} B, C_{3}:=C_{2} B$, and

$$
\begin{array}{ll}
D_{1}:=\left(A\left(C_{2} C_{3} C_{2}^{2} C_{3}^{2}\right)^{5}\right)^{4}, & D_{4}:=\left(A\left(B^{2} C_{3}^{2} C_{2} C_{1}\right)^{5}\right)^{2}, \\
D_{2}:=\left(A\left(B^{3} C_{1}\right)^{5}\right)^{4}, & D_{5}:=\left(A\left(\left(\left(C_{1} C_{2}\right)^{2} C_{2}\right)^{3} C_{1} C_{2}^{2}\right)^{5}\right)^{5}, \\
D_{3}:=\left(A\left(\left(C_{1} C_{2}\right)^{5} C_{2}\right)^{6}\right)^{6}, & D_{6}:=\left(A\left(B^{2}\left(C_{3} C_{2} C_{3}\right)^{2} C_{2} C_{3}\left(C_{3} C_{2} C_{3}\right)^{3} C_{2} C_{1}\right)^{5}\right)^{4} .
\end{array}
$$

It is then shown, again using GAP, that $\left\langle D_{1}, D_{2}, D_{3}, D_{4}, D_{5}, D_{6}\right\rangle$ is a subgroup of the centralizer of $A$ in $S u z$, having order 3317760 , hence $M_{4}$ is generated by these elements.

Next we consider the matrix representation corresponding to Suz.p1782. Its entries are rational integers, hence they can be reduced modulo the rational prime 2 , giving a matrix representation of $S u z$ over $G F(2)$. Now the MeatAxe is used to 'chop' this module. Especially, we find a constituent $142_{G F(2)}$ of dimension 142. Considering the restriction of $142_{G F(2)}$ to $M_{4}$, the MeatAxe shows that there is exactly one submodule isomorphic to the trivial $M_{4}$-module. Clearly, the orbit of this vector under the action of Suz has length 135135, which gives us Suz.p135135.

7.4. Our next task is to find a suitable condensation subgroup, which in our case has to be a group of order prime to 3 . The maximal normal subgroup $O_{2}:=2_{-}^{1+6}$ of $M_{4}$, an extraspecial group of order 128, turns out to be suitable for our purposes. We define $E_{1}:=D_{1} D_{2} D_{3}, E_{2}:=D_{4} D_{5}, F_{1}:=E_{1} E_{2}, F_{2}:=F_{1} E_{2}, F_{3}:=F_{2} E_{2}$, $F_{4}:=E_{1} F_{1}$, and

$$
\begin{array}{lll}
G_{1}:=\left(F_{2}^{2} F_{3}\right)^{2}, & G_{3}:=\left(F_{4}^{2} F_{1} F_{4}^{3} F_{1}\right)^{2}, & G_{5}:=\left(F_{4}^{2} F_{1} F_{4}^{3} F_{1} F_{4} F_{1}\right)^{6}, \\
G_{2}:=\left(F_{2} F_{3}^{2} F_{2}^{2} F_{3}\right)^{2}, & G_{4}:=\left(F_{4}^{4} F_{1}\right)^{6}, & G_{6}:=\left(F_{1} F_{2}\left(F_{1} F_{2}^{2}\right)^{2}\left(F_{1} F_{2}\right)^{3} F_{2}\right)^{6} .
\end{array}
$$

It is seen using GAP that we have $O_{2}=\left\langle G_{1}, G_{2}, G_{3}, G_{4}, G_{5}, G_{6}\right\rangle$.

In the sequel let $e:=e_{\mathrm{O}_{2}}$ denote the idempotent corresponding to $\mathrm{O}_{2}$. We consider condensed modules as modules for the algebra generated by $\{e X e, e Y e, e Z e\}$, where $X:=C_{1}^{2} C_{2}, Y:=C_{1}^{4} C_{2}$, and $Z:=C_{1} C_{2} C_{1}^{2} C_{2}^{2}$. This algebra, which in the following is referred to as the condensation algebra, is contained in $e F G e$, but of course we cannot be sure that it is equal to it.

7.5. Finally, we determine the dimension of the condensed modules corresponding to the ordinary characters of $S u z$ up to degree 66560 , by computing the scalar products of the restrictions of these characters to the subgroup $\mathrm{O}_{2}$ with the trivial character of $\mathrm{O}_{2}$. Using GAP, we find that $\mathrm{O}_{2}$ contains 55 elements of order 2 and 72 elements of order 4. Using the fusion of $M_{4}$ into $S u z$ determined in Section 3 and the factor fusion from Section 3.3, the scalar products can be computed, the result is given in Table 15. From these data we compute the dimensions of the condensed modules corresponding to the Brauer characters in $B S$ given in Section 4.12, which is used from Section 8.2 on. They can be found in Table 16. Note that in Section 8.1 we use $B S$ given in Section 4.10 instead. The condensed module corresponding to the Brauer character 935 in there has dimension 19.

\section{Analyzing Representations}

8.1. Suz.p1782. The aim of this section is to show the existence of 649 , as was asserted in Section 4.11, and to find the isomorphism types of the condensed modules 
TABLE 15. Ordinary characters and the dimensions of the corresponding condensed modules

\begin{tabular}{r|rr|rr|r}
1 & 1 & 10725 & 180 & $25025_{3}$ & 100 \\
143 & 16 & 12012 & 234 & 40040 & 490 \\
364 & 24 & 14300 & 195 & $50050_{1}$ & 315 \\
780 & 27 & $15015_{1}$ & 216 & $50050_{2}$ & 315 \\
1001 & 26 & $15015_{2}$ & 216 & 54054 & 612 \\
3432 & 48 & 15795 & 243 & 64064 & 564 \\
$5005_{1}$ & 70 & 18954 & 162 & $64350_{1}$ & 510 \\
$5005_{2}$ & 70 & $25025_{1}$ & 215 & $64350_{2}$ & 510 \\
5940 & 135 & $25025_{2}$ & 100 & 66560 & 520
\end{tabular}

TABLE 16. Brauer characters and the dimensions of the corresponding condensed modules

\begin{tabular}{r|rr|rr|r}
1 & 1 & 429 & 7 & 8436 & 68 \\
64 & 5 & 649 & 5 & 14730 & 84 \\
78 & 10 & 1938 & 10 & 19513 & 40 \\
286 & 14 & 2925 & 45 & 32967 & 297
\end{tabular}

TABLE 17. Constituents of the condensed representation Suz.p1782

\begin{tabular}{r|r|r|r}
1 & 1 & 1 & $1 a$ \\
\hline 780 & 1 & 1 & $1 a$ \\
& 64 & 1 & $5 a$ \\
& 286 & 1 & $14 a$ \\
& 429 & 1 & $7 a$ \\
\hline 1001 & 1 & 2 & $1 a$ \\
& 64 & 1 & $5 a$ \\
& 935 & 1 & $5 b+14 a$
\end{tabular}

corresponding to the 3-modular constituents of Suz.p1782. The decomposition of the ordinary constituents of Suz.p1782 into BS given in Section 4.10 is shown in Table 17. In the first column we give the ordinary constituents and in the second and third columns their 3-modular constituents and the corresponding multiplicities.

To show the existence of 649 , we consider the matrix representation corresponding to Suz.p1782. Its entries are rational integers, hence they can be reduced modulo the rational prime 3. Then this module is 'chopped', giving 649 explicitly as one of the constituents. The explicit matrices are needed in Section 8.5. We remark that it is possible to show the mere existence of 649 by analyzing the submodule lattice of the condensed module corresponding to Suz.p1782, without 'chopping' the permutation module explicitly.

We now use the MeatAxe to construct and 'chop' the condensed module corresponding to Suz.p1782 with respect to the subgroup $\mathrm{O}_{2}$. It is found to have 
dimension 54 and its constituents as a module for the condensation algebra are $1 a^{4}, 5 a^{2}, 5 b, 7 a, 14 a^{2}$, where the exponents denote multiplicities. Counting multiplicities and dimensions of these constituents, we find that the constituents 1,64 , 286,429 , and 649 condense onto $1 a, 5 a, 14 a, 7 a$, and $5 b$, respectively. This correspondence is given in the forth column of Table 17 .

8.2. Suz.p135135. We now determine $x, y$, and $z$, see Section 4.13, by an analysis of the condensed module $M$ corresponding to Suz.p135135. The constituents of $M$, which has dimension 1319 , as a module for the condensation algebra, as they are found by the MeatAxe, are as follows:

$$
1 a^{14}, 5 a^{10}, 5 b^{7}, 7 a^{6}, 10 a^{5}, 10 b^{2}, 14 a^{11}, 35 a^{1}, 45 a^{3}, 68 a^{4}, 84 a^{2}, 91 a^{2}, 162 a^{1} .
$$

The ordinary constituents of Suz.p 135135 and their decomposition into $B S$ given in Section 4.12 is shown in Table 18. The ordinary constituent 18954 is the only one not belonging to the principal block.

Comparing dimensions, it follows that 18954 corresponds to the constituent $162 a$. Therefore $M$ has a unique direct summand $M_{162}$ isomorphic to $162 a$. We use the MeatAxe to find a peakword, see [10], for $162 a$. The kernel of its action on $M$ is a subspace of $M_{162}$. It is now a standard application of the MeatAxe to compute the quotient module $M_{p b}:=M / M_{162}$. In the sequel we examine $M_{p b}$ in more detail.

From the results in Section 8.1 we know that the multiplicity of the constituent $14 a$ in $M_{p b}$ is greater than or equal to the multiplicity of the irreducible Brauer character 286 in the decomposition of the permutation character of Suz.p135135. Now a comparison of multiplicities shows that 14730 cannot contain another 286, hence we have $x=0$. An analogous argument using $5 b$, which corresponds to 649 , shows that also $y=0$. Hence $\varphi_{11}=14730$ is irreducible. We remark that this result could also be deduced using the condensed tensor product module which is considered in Section 8.5.

8.3. Now we turn our attention to the determination of $z$. Comparing dimensions and multiplicities it is straightforward that $91 a, 84 a, 68 a$, and $45 a$ correspond to $4785,14730,8436$, and 2925 , respectively. Hence $35 a$ is a constituent of the condensed module corresponding to 19513. Now 19513 condenses onto a module of dimension 40. Counting multiplicities of constituents which have not yet been shown to correspond to an irreducible Brauer character, we find that 19513 condenses onto a module having the constituents $35 a, 5 a$.

The MeatAxe finds a peakword for $5 a$. Then each minimal $5 a$-local submodule of $M_{p b}$ is generated by an element of the kernel of its action on $M_{p b}$, as follows from the results in [10]. We now look for those of them which have a dimension less than 44 ; this bound becomes meaningful, as soon as we have defined the submodule $U_{43}$ a few lines below. The MeatAxe shows that there are exactly four such submodules. One submodule $L_{6}$ of dimension 6 and three submodules $L_{27}^{1}, L_{27}^{2}$, and $L_{27}^{3}$ of dimension 27. Furthermore, each of the $L_{6}$ and $L_{27}^{1,2,3}$ contain the constituent $5 a$ exactly once, and we have $L_{6} \cap L_{27}^{1}=L_{6} \cap L_{27}^{2}=L_{6} \cap L_{27}^{3}$, being of dimension 1 .

By the Zassenhaus Theorem 6.5, there is an $e F G e$-submodule $U_{16}$ of $M_{p b}$ of dimension 16 corresponding to the ordinary constituent 143 of Suz.p135135. Since all the irreducible 3-modular constituents of 143 are known and 64 is one of them, $U_{16}$ has $5 a$ as one of its constituents. Hence by Remark 6.3, it follows that $\tilde{L}_{1}:=L_{6} \cdot e F G e$ is a $5 a$-local $e F G e$-submodule of $U_{16}$. Again by the Zassenhaus Theorem, there exists an $e F G e$-submodule $U_{43}$ of dimension 43 which contains $U_{16}$ 
TABle 18. Constituents of the condensed representation Suz.p135135

\begin{tabular}{|c|c|c|c|}
\hline 1 & 1 & 1 & $1 a$ \\
\hline \multirow{3}{*}{143} & 1 & 1 & $1 a$ \\
\hline & 64 & 1 & $5 a$ \\
\hline & 78 & 1 & $10 a$ \\
\hline \multirow[t]{4}{*}{780} & 1 & 1 & $1 a$ \\
\hline & 64 & 1 & $5 a$ \\
\hline & 286 & 1 & $14 a$ \\
\hline & 429 & 1 & $7 a$ \\
\hline \multirow[t]{6}{*}{3432} & 1 & 2 & $1 a$ \\
\hline & 64 & 2 & $5 a$ \\
\hline & 286 & 1 & $14 a$ \\
\hline & 429 & 1 & $7 a$ \\
\hline & 649 & 1 & $5 b$ \\
\hline & 1938 & 1 & $10 b$ \\
\hline \multirow[t]{7}{*}{5940} & 1 & 2 & $1 a$ \\
\hline & 64 & 1 & $5 a$ \\
\hline & 78 & 1 & $10 a$ \\
\hline & 286 & 4 & $14 a$ \\
\hline & 429 & 1 & $7 a$ \\
\hline & 649 & 2 & $5 b$ \\
\hline & 2925 & 1 & $45 a$ \\
\hline \multirow[t]{7}{*}{14300} & 1 & 2 & $1 a$ \\
\hline & 64 & 1 & $5 a$ \\
\hline & 78 & 1 & $10 a$ \\
\hline & 286 & 1 & $14 a$ \\
\hline & 649 & 1 & $5 b$ \\
\hline & 4785 & 1 & $91 a$ \\
\hline & 8436 & 1 & $68 a$ \\
\hline \multirow[t]{8}{*}{$25025_{1}$} & 1 & 3 & $1 a$ \\
\hline & 64 & 2 & $5 a$ \\
\hline & 78 & 1 & $10 a$ \\
\hline & 286 & 2 & $14 a$ \\
\hline & 429 & 1 & $7 a$ \\
\hline & 649 & 1 & $5 b$ \\
\hline & 8436 & 1 & $68 a$ \\
\hline & 14730 & 1 & $84 a$ \\
\hline \multirow[t]{12}{*}{66560} & 1 & 2 & $1 a$ \\
\hline & 64 & 1 & $5 a$ \\
\hline & 78 & 1 & $10 a$ \\
\hline & 286 & 2 & $14 a$ \\
\hline & 429 & 2 & $7 a$ \\
\hline & 649 & 2 & $5 b$ \\
\hline & 1938 & 1 & $10 b$ \\
\hline & 2925 & 2 & $45 a$ \\
\hline & 4785 & 1 & $91 a$ \\
\hline & 8436 & 2 & $68 a$ \\
\hline & 14730 & 1 & $84 a$ \\
\hline & 19513 & 1 & $5 a+35 a$ \\
\hline 18954 & 18954 & 1 & $162 a$ \\
\hline
\end{tabular}


TABLE 19. Constituents of the condensed representation Suz.p22880

\begin{tabular}{r|r|r|r}
1 & 1 & 1 & $1 a$ \\
\hline 364 & 78 & 1 & $10 a$ \\
& 286 & 1 & $14 a$ \\
\hline 780 & 1 & 1 & $1 a$ \\
& 64 & 1 & $5 a$ \\
& 286 & 1 & $14 a$ \\
& 429 & 1 & $7 a$ \\
\hline 5940 & 1 & 2 & $1 a$ \\
& 64 & 1 & $5 a$ \\
& 78 & 1 & $10 a$ \\
& 286 & 4 & $14 a$ \\
& 429 & 1 & $7 a$ \\
& 649 & 2 & $5 b$ \\
& 2925 & 1 & $45 a$ \\
\hline 15795 & 15795 & 1 & $243 a$
\end{tabular}

and corresponds to the ordinary character $143+780$. Now the 3 -modular constituents of 780 are also known and 64 is again one of them, hence for one of the $L_{27}^{i}, L_{27}^{1}$ say, we get $\tilde{L}_{2}:=L_{27}^{1} \cdot e F G e$ as a $5 a$-local $e F G e$-submodule of $U_{43}$. Clearly, we have $\tilde{L}_{1} \neq \tilde{L}_{2}$.

Using the Zassenhaus Theorem once again, we see that $M_{p b}$ contains an eFGesubmodule $U_{520}$ of dimension 520 which corresponds to the ordinary constituent 66560 of Suz.p135135. The MeatAxe finds a peakword for the constituent $35 a$. The kernel of its action on $M_{p b}$ is of dimension 1, and it generates a $35 a$-local submodule $L_{448}$ of $M_{p b}$ of dimension 448. Since $U_{520}$ has a constituent $35 a$, we have $L_{448} \leq$ $U_{520}$. Now the MeatAxe shows that $L_{6}+L_{27}^{1} \leq L_{448}$. Hence we have $\tilde{L}_{1}+\tilde{L}_{2} \leq U_{520}$. Therefore, the ordinary character 66560 contains the irreducible Brauer character 64 at least twice. Hence we have $z=1$ and $\varphi_{12}=19513-64=19449$ as an irreducible Brauer character.

8.4. Suz.p22880. Before we finally determine $w$, see Section 4.13, we have to do some preparatory work. The ordinary constituents of the permutation character of Suz.p22880 are given in Table 19. The ordinary character 15795 belongs to the second block and reduces irreducibly modulo 3, as was shown in Section 5. Hence all the 3-modular constituents of Suz.p22880 are known.

The condensed module $N$ corresponding to Suz.p22880 has dimension 430. Using the MeatAxe we find its constituents as a module for the condensation algebra: $1 a^{4}, 5 a^{2}, 5 b^{2}, 7 a^{2}, 10 a^{2}, 14 a^{6}, 45 a, 243 a$. Counting dimensions and multiplicities, we find that $10 a$ is the constituent the irreducible Brauer character 78 condenses to.

Now again by the Zassenhaus Theorem 6.5, there is an eFGe-submodule $U_{24}$ of $N$ of dimension 24, which corresponds to the ordinary constituent 364 of the permutation character. Using the peakword technique mentioned earlier, it is shown that there is exactly one $10 a$-local submodule $L \leq N$ of dimension not exceeding 24. Since $L$ has dimension 24 , we have $U_{24}=L$. The methods explained in [10] also yield a generating vector $v$ for $L$. Now the condensed module $V e$ is a subset of $V$. Hence $v$, considered as an element of $V$, generates a submodule of 
the permutation module of dimension 364, which is a 3 -modular reduction of the ordinary irreducible module of dimension 364. It is now a standard application of the MeatAxe to compute the action of the chosen generators for $S u z$ on this submodule, and to obtain its constituent 78 .

8.5. Suz.78 $\otimes 649$. Now we are ready to determine $w$, see Section 4.13 , by applying the TensorCondense package. A short comment on the application of TensorCondense is in order. A crucial step in the procedure is to find suitable symmetry bases, see [11], for the tensor factors 78 and 649. The package provides two different methods to find such bases, the peakword method and the $\gamma$-operator method, none of which is well-suited for our case. This is because our condensation subgroup $\mathrm{O}_{2}$ has 64 different linear representations. But in the case of a linear representation the corresponding part of a suitable symmetry basis can be found by explicitly computing simultaneous eigenspaces for the generators of the condensation subgroup. This is done by concatenating a few of the standard applications of the MeatAxe.

The Brauer character of the tensor product $78 \otimes 649$ decomposes as $78 \otimes 649=$ $2925+14730+32967$ into $B S$ given in Section 4.12. Hence we show that the corresponding condensed module has composition length 3 as a module for the condensation algebra. It then follows that $w=0$, i. e., $\varphi_{13}=32967$ is irreducible. Note that this gives an alternative proof of the irreducibility of $\varphi_{11}=14730$, see Section 8.2.

Now applying TensorCondense using the explicit matrices for 78 and 649 constructed in Sections 8.4 and 8.1 yields the condensed module, which is of dimension 426. The standard MeatAxe shows that it has the constituents $45 a, 84 a, 297 a$ as a module for the condensation algebra, and we are done.

\section{REFERENCES}

[1] D. Benson: Representations and Cohomology, vol. I, Cambridge, 1991.

[2] D. Benson, J. Carlson: Nilpotent Elements in the Green Ring, J. Algebra 104:329-350, 1986.

[3] J. Conway, R. Curtis, S. Norton, R. Parker, R. Wilson: Atlas of Finite Groups, Clarendon, 1985.

[4] W. Feit: The representation theory of finite groups, North-Holland, 1982.

[5] G. Hiss, C. Jansen, K. Lux, R. Parker: Computational Modular Character Theory, Preprint, 1992.

[6] G. Hiss, K. Lux: Brauer Trees of Sporadic Groups, Clarendon, 1989.

[7] C. Jansen: Ein Atlas 3-modularer Charaktertafeln, Dissertation, RWTH Aachen, 1995.

[8] C. Jansen, K. Lux, R. Parker, R. Wilson: An Atlas of Brauer Characters, Oxford University Press, 1995.

[9] P. Landrock: Finite Group Algebras and Their Modules, Cambridge, 1983.

[10] K. Lux, J. Müller, M. Ringe: Peakword Condensation and Submodule Lattices, J. Symb. Comp. 17:529-544, 1994.

[11] K. Lux, M. Wiegelmann: Condensing Tensor Product Modules, in preparation.

[12] J. Müller: 5-modulare Zerlegungszahlen für die sporadische einfache Gruppe Co 3 , Diplomarbeit, RWTH Aachen, 1991.

[13] R. Parker: The Computer Calculation of Modular Characters, in Atkinson (ed.): Computational Group Theory, 1984.

[14] M. Ringe: The C-MeatAxe, Documentation, RWTH Aachen, 1992.

[15] A. Ryba: Computer Condensation of Modular Representations, J. Symb. Comp. 9:591-600, 1990.

[16] M. Schönert (ed.): GAP-3.4 Manual, RWTH Aachen, 1994.

[17] M. Wiegelmann: Fixpunktkondensation von Tensorproduktmoduln, Diplomarbeit, RWTH Aachen, 1994. 
(Christoph Jansen) Lehrstuhl D für Mathematik, RWTH Aachen, Templergraben 64, D-52056 AACHEN

E-mail address: cjansen@math.rwth-aachen.de

(Jürgen Müller) InterdisZiplinÄres Zentrum Für Wissenschaftliches Rechnen, UniverSität Heidelberg, Im Neuenheimer Feld 368, D-69120 HeidelberG

E-mail address: jmueller@erato.iwr.uni-heidelberg.de 